\title{
Relations entre organisation du travail et taille des exploitations laitières : une étude à Moc Chau (Vietnam)*
}

\author{
Nathalie Hostiou ${ }^{1}$ \\ Benoit Dedieu ${ }^{1}$ \\ Sophie Madelrieux ${ }^{2}$ \\ Duy Khanh Pham ${ }^{3}$ \\ Binh Vu Trong ${ }^{3}$ \\ 1 Inra \\ Umr Métafort \\ 63122 Saint Genès Champanelle \\ France \\ <nhostiou@clermont.inra.fr> \\ $<$ dedieu@clermont.inra.fr> \\ 2 Cemagref \\ 2 , rue de la Papeterie \\ 38402 St Martin \\ St Martin d'Hères \\ France \\ <sophie.madelrieux@cemagref.fr> \\ 3 RUDEC \\ 69 Kim Ma Thuong \\ Hanoi \\ Viet Nam \\ <duykhanh131180@yahoo.com> \\ <trongbinh@fpt.vn>
}

\begin{abstract}
Résumé
Au Vietnam, les politiques agricoles visent à développer la production laitière pour accroitre l'autonomie du pays. Cependant, les objectifs nationaux sont loin d'être atteints. Le travail est perçu comme une des contraintes à l'accroissement de la production dans les exploitations familiales. L'objectif de cet article est d'explorer les relations entre les durées et les formes d'organisation du travail en lien avec la taille des troupeaux de façon à discuter ce qu'impliquerait une rationalisation du travail par l'agrandissement des cheptels. Une enquête a été réalisée auprès de neuf exploitants de la région de Moc Chau associant deux méthodes d'analyse de l'organisation du travail. L'analyse des données a consisté à extraire des variables pour identifier : i) les relations entre taille des troupeaux et des surfaces, durées et forme d'organisation du travail, ii) des types d'organisation du travail. Nos résultats montrent que le volume de travail d'astreinte est plus élevé dans les plus grands troupeaux, mais il est également plus efficient (en heures/ vache/an). Trois types d'organisation ont été identifiés. Par exemple, dans les plus grandes exploitations, présentant un niveau d'intensification plus élevé, les éleveurs allègent leur travail via la mécanisation de tâches d'astreinte (améliorant ainsi l'efficience du travail d'astreinte par vache) et par l'emploi de salariés permanents. Dans un contexte où la conduite technique est normée par l'aval, les principales sources d'ajustement du travail sont le niveau d'équipement, l'emploi de salarié et l'arrêt de l'activité non agricole pendant les périodes de pointes. Nous concluons sur des pistes d'accompagnement telles que le salariat et la mécanisation pour appuyer le développement de l'activité laitière.
\end{abstract}

Mots clés : bovin ; exploitation agricole familiale ; exploitation laitière ; organisation du travail.

Thèmes : agriculture familiale ; économie et développement rural ; mécanisation et aménagement rural.

\section{Abstract \\ Relationships between work organisation and size of dairy farms: A study in Moc Chau (Vietnam)}

In Vietnam, farming policies are aimed at developing milk production to increase the country's self-sufficiency. National objectives, which are far from being reached, bring into question factors that limit greater dairy production. Work is perceived as one of the constraints to the development of this activity on the family dairy farms. The aim of this article is to explore the relationships between work duration, forms of work organisation and the size of herds, in order to discuss what would be involved in rationalising the work by increasing herd size. A survey was carried out with nine farmers in the Moc Chau region, combining two work methods. Data analysis consisted in extracting variables to identify i) relationships between herd and area size, duration and forms of work organisation, ii) types of work organisation. Our results point out that the total daily work routine is longer in the biggest herds, but it is also more efficient (hours/cow/ year). Three types of organisation have been identified. For example, in the largest farms, with a higher level of intensification (productivity per cow), farmers reduce their

\footnotetext{
* Pour citer cet article: Hostiou N, Dedieu B, Madelrieux S, Khanh Pham D, Vu Trong B. Relations entre organisation du travail et taille des exploitations laitières: une étude à Moc Chau (Vietnam). Cah Agric 2010 ; 19 : 323-30. DOI : 10.1684/agr.2010.0404. 
work load by mechanising routine tasks and employing permanent hired workers. In a context in which technical management is standardised, the main sources of work adjustment are the level of equipment, the employment of hired workers and the cessation of the non-farming activity during workload periods. We conclude on ways of guiding and supporting farms in the development of their dairy activity, such as the employment of workers and mechanisation.

Key words: cattle; dairy farm; family farms; work organization.

Subjects: family farming; economy and rural development; mechanization and rural planning.

e Vietnam cherche à développer sa production laitière pour accrô̂tre son autonomie, en augmentant le nombre de vaches et d'exploitations familiales (Suzuki et al., 2006), cependant avec peu de succès. Dans les exploitations familiales de petite dimension, la faible rationalisation du travail est considérée comme une des sources de blocage pour accroître la production laitière (Martin et al., 2004 ; Cramb et al., 2004). Les exploitations utilisent avant tout de la main-d'œuvre familiale, peu nombreuse et souvent engagée dans la pluriactivité (Cramb et al., 2004). Le travail est manuel, avec un niveau de mécanisation faible, voire inexistant. De ce fait, la mise en œuvre d'itinéraires techniques sophistiqués, fondés sur l'ensilage de maïs et l'affouragement en vert, induit des durées de travail très élevées (Martin et al., 2004). L'agrandissement des troupeaux laitiers apparaît comme une option possible pour rationaliser le travail, dans la mesure où il permettrait d'accéder à des économies d'échelle et à de la mécanisation. Cependant, localement, il n'existe pas de connaissance disponible sur l'organisation du travail en milieu paysan et sur la façon dont le travail évolue avec la taille du cheptel. Dans le cadre du projet ADDTrans (transformation de l'élevage et dynamique des espaces), une étude a été effectuée pour tester l'adaptabilité de méthodes d'analyse de l'organisation du travail aux réalités et aux spécificités vietnamiennes, et produire les premières connaissances sur le travail en élevage laitier dans ce pays. Sur la base d'un petit échantillon d'exploitations laitières, l'objectif de l'article est d'explorer comment la taille du troupeau laitier pourrait marquer les formes d'organisation du travail et les temps de travaux. Il s'agit d'identifier des pistes pour accompagner l'augmentation de la production laitière par l'agrandissement des troupeaux. Cette étude, tout à fait originale dans ce pays, a été réalisée dans une région de montagne au Nord du Vietnam (Moc Chau), orientée vers l'activité laitière.

\section{Matériel et méthode}

Le district de Moc Chau (2050' de latitude $\mathrm{N}$; 104³8' de longitude E) se situe à 200 kilomètres au nord-ouest de Hanoi. Une ferme d'État y a été créée en 1975 pour la production de lait et de thé. Depuis les années 1990, la "Compagnie laitière " (ancienne ferme d'état privatisée) organise le territoire et l'élevage laitier familial. Les éleveurs, souvent anciens ouvriers de la ferme, ont obtenu des vaches et ont loué les terres. En 2005, le cheptel était de 3300 vaches laitières Prim Holstein pour 504 exploitations familiales. Les éleveurs dépendent fortement de la coopérative qui définit les pratiques d'élevage, contrôle les effectifs d'animaux, fournit les services techniques et vétérinaires, achète le lait. Les vaches sont conduites en zéro pâturage toute l'année avec un affouragement à l'auge issu d'une coupe quotidienne de fourrages tropicaux en été et une distribution d'ensilage de mais et de foin en hiver. Ce modèle technique permet un niveau de chargement élevé (trois à six têtes par hectare) ainsi que la généralisation des cultures fourragères. Il est a priori très demandeur en travail tout au long de l'année. La diminution du nombre d'exploitations observée depuis les dernières années ouvre des perspectives aux éleveurs pour accroitre leur cheptel. Une enquête a été réalisée dans neuf exploitations, choisies pour couvrir la diversité de taille d'élevage laitier (tableau 1) que l'on retrouve dans le bassin de Moc Chau. Les cheptels étudiés varient de 3 à 32 têtes - vaches et génisses de plus de 1 an, pour une production annuelle de 6 à 85 tonnes de lait et une surface de 0,5 à 7 hectares. En moyenne, les exploitations du bassin de Moc Chau comptent 6,48 vaches pour 1,79 hectare de surface agricole utile (SAU), mais leur diversité est forte : $52 \%$ des exploitations ont moins de 3 têtes de bétail et 8,5\% des exploitations en ont plus de 10. Dans l'échantillon, sept exploitations sont gérées par des couples, et deux par des exploitants seuls. Quatre emploient des salariés permanents. Six exploitations sont spécialisées et trois associent une activité non agricole (vente de produits vétérinaires par exemple).

Une enquête d'une demi-journée a été réalisée avec les chefs d'exploitation. Elle associait les principes de deux méthodes d'analyse du travail proposées en zootechnie des systèmes d'élevage: Bilan travail (Dedieu et al., 2006) et Atelage (Madelrieux et Dedieu, 2008). Leurs finalités opérationnelles sont, d'une part, d'accompagner les éleveurs dans leur réflexion sur les évolutions de leur système d'exploitation et, d'autre part, d'évaluer les conséquences de changements techniques sur l'organisation du travail. Dans un premier temps, l'enquête reprend les bases de l'approche globale de l'exploitation, puis, sur la base d'un calendrier annuel, elle décrit, période par période, l'organisation du travail (le "qui fait quoi " et la durée du travail pour les différents travailleurs). Elle permet également de rendre compte de l'adaptation de cette organisation selon l'évolution du calendrier technique (été/hiver), de la disponibilité de la maind'œuvre (présence/absence de travailleurs) et des interactions entre activités agricoles et non agricoles. Pour traiter du contenu du travail (le "quoi"), l'enquêteur distingue deux types de travaux selon leur capacité à être différés d'une journée à l'autre. Le travail 
Tableau 1. Caractéristiques des neuf exploitations laitières à Moc Chau.

Table 1. Characteristics of the nine dairy farms in Moc Chau.

\begin{tabular}{|c|c|c|c|c|c|c|c|c|c|}
\hline Élevage & $\begin{array}{l}\text { Surface } \\
\text { (hectares) }\end{array}$ & $\begin{array}{c}\mathrm{Nb} \\
\text { de têtes }\end{array}$ & $\begin{array}{c}\mathrm{Nb} \\
\text { de vaches }\end{array}$ & $\begin{array}{l}\text { Lait/an } \\
\text { (tonne) }\end{array}$ & $\begin{array}{l}\text { Production } \\
\text { lait/vache/an } \\
\text { (litres) }\end{array}$ & $\begin{array}{c}\text { Charge } \\
\text { animale/ha } \\
\text { de SAU* }\end{array}$ & $\begin{array}{l}\text { Activité } \\
\text { non agricole }\end{array}$ & $\begin{array}{l}\text { Cellule } \\
\text { de base } \\
\text { (CB) }\end{array}$ & $\begin{array}{c}\text { Nombre } \\
\text { de salariés } \\
\text { permanents }\end{array}$ \\
\hline $\mathrm{HP}$ & 7 & 32 & 20 & 85 & 4300 & 3,7 & - & Couple & 1 \\
\hline TY & 2 & 9 & 7 & 35 & 5000 & 4,0 & - & Mme & 1 \\
\hline VN & 4,7 & 32 & 19 & 75 & 3900 & 5,4 & - & Couple & 2 \\
\hline DE & 4 & 15 & 10 & 50 & 5000 & 3,1 & - & Couple & 1 \\
\hline $\mathrm{CN}$ & 2 & 10 & 8 & 40 & 5000 & 4,5 & - & Couple & 0 \\
\hline CG & 3,4 & 9 & 7 & 30 & 4300 & 2,4 & Collecte de thé & Couple & 0 \\
\hline DC & 1,8 & 5 & 5 & 22 & 4000 & 2,8 & - & Couple & 0 \\
\hline $\mathrm{HG}$ & 1,2 & 4 & 4 & 8 & 2000 & 3,3 & $\begin{array}{l}\text { Médecine } \\
\text { traditionnelle }\end{array}$ & Couple & 0 \\
\hline $\mathrm{HC}$ & 0,5 & 3 & 3 & 6 & 2000 & 6,0 & $\begin{array}{l}\text { Vente produits } \\
\text { vétérinaires }\end{array}$ & Mme & 0 \\
\hline
\end{tabular}

* SAU : surface agricole utile.

d'astreinte est à réaliser quasi quotidiennement, il concerne généralement les soins journaliers aux animaux (traite, alimentation, curage...). Il est quantifié en heures par jour. Le travail de saison concerne des tâches susceptibles d'être différées et/ou concentrables sur une période donnée. Il comprend les travaux liés aux activités agricoles: troupeau, cultures, surfaces fourragères. Il est quantifié en jours par an. Pour traiter de la main-d'oeuvre (le " qui "), l'enquêteur distingue la cellule de base, c'est-à-dire les travailleurs pour qui l'activité agricole est prépondérante en temps et en revenu et qui organisent le travail sur l'exploitation - exploitant, couple d'exploitants, associés - et la main-d'œuvre hors cellule de base qui comprend des bénévoles, des salariés, un recours à l'entraide et à l'entreprise. Les temps de travaux ne sont pas enregistrés, mais estimés par l'éleveur lors de l'enquête. Finalement, les données recueillies par entretien permettent: i) de qualifier les formes d'organisation du travail d'astreinte - qui représentent, au cours de l'année, les évolutions de la main-d'œuvre et du contenu du travail - (tableau 2); ii) de quantifier les durées du travail d'astreinte et de saison ; iii) de quantifier la part du travail réalisée par la cellule de base et les autres catégories de travailleurs. Les données sur la production globale du troupeau ont été obtenues de manière déclarative auprès des éleveurs. L'analyse des données a été réalisée en trois étapes:

- mise en forme des données pour chaque exploitation ;
- sélection de variables caractérisant les durées du travail (deux variables) et les formes d'organisation du travail (quatre variables) (tableau 3);

- identification de types d'organisation du travail. Cette étape a mobilisé une méthode particulière d'analyse graphique (Bertin, 1977) qui amplifie la cognition visuelle. Nous avons créé une matrice avec les exploitations en colonne, et les différentes variables et leurs modalités en ligne (tableau 4). Nous avons rapproché les exploitations présentant des modalités similaires, repérées visuellement, pour constituer des groupes (Girard et al., 2001).

\section{Résultats}

\section{Niveau d'intensification, durées et efficience du travail}

Les exploitations de notre échantillon présentent des niveaux d'intensification variables (tableau 1). Il ne semble pas y avoir de relation entre la charge animale par hectare et la taille du troupeau. Nous avons pu observer un lien entre le niveau d'intensification (productivité par vache) et l'agrandissement des cheptels. En effet, les exploitations avec les plus grands cheptels ont des niveaux de productivité par vache plus élevés (tableau 1).

Le travail d'astreinte (TA) total dans l'année varie de 1494 à 6024 heures, soit de 4 à $17 \mathrm{~h}$ par jour (tableau 5). Il comprend des tâches quotidiennes avec le troupeau (traite, vente du lait, coupe d'herbe, distribution des aliments, curage de la stabulation) et la vente du lait (déplacement vers le lieu de collecte). La durée annuelle du travail d'astreinte est plus élevée dans les plus grands troupeaux, mais sans relation linéaire robuste avec l'effectif. Cependant, les plus grandes exploitations ont une meilleure efficience du travail d'astreinte, exprimée en heures par an (TA/vache/an). Par exemple, les exploitations avec des troupeaux de 20 vaches laitières $(\mathrm{HP}, \mathrm{VN})$ ont un travail d'astreinte inférieur à 320 heures/vache/ an. Dans les élevages avec de plus petits troupeaux (DC, HG, HC), le temps de travail d'astreinte est de plus de 500 heures/ vache/an. Dans les plus grands élevages, la mécanisation de la traite avec un pot trayeur et celle de la coupe de l'herbe avec un rotofil permettent d'améliorer l'efficience du travail (tableau 5). Dans ces exploitations, une part importante du travail d'astreinte est déléguée à des salariés permanents ou à des aides bénévoles (39 à $65 \%$ du travail d'astreinte est réalisé par la main-d'œuvre hors cellule de base). Des tâches parmi les plus pénibles physiquement, à savoir le curage de la stabulation et/ou la coupe quotidienne de l'herbe, leur sont confiées.

Le travail de saison est consacré à la surface fourragère (labour, foin, ensilage de maïs, etc.), et est réalisé sans aucune mécanisation. Il est fortement variable entre les exploitations de l'échantillon (34 à 199 jours par an) (tableau 5). Il existe 
Tableau 2. Un calendrier de main-d'œuvre et technique en lien avec les durées et les formes d'organisation du travail (exploitation HC).

Table 2. A workforce and technical calendar linked with duration and forms of work organization (farm HC).

\begin{tabular}{|c|c|c|c|c|c|c|c|c|c|c|c|c|c|}
\hline & & D & $\mathbf{J}$ & $\mathbf{F}$ & $\mathbf{M}$ & A & $\mathbf{M}$ & $\mathbf{J}$ & Jt & A & $\mathbf{S}$ & 0 & $\mathbf{N}$ \\
\hline \multirow[t]{2}{*}{ Main-d'œuvre } & $\begin{array}{l}\text { Cellule } \\
\text { de base }\end{array}$ & \multicolumn{6}{|c|}{ Mme HC tous les jours, M. HC double actif } & \multicolumn{3}{|c|}{ M. et Mme tous les jours } & \multicolumn{3}{|c|}{$\begin{array}{l}\text { Mme HC tous les } \\
\text { jours, } \\
\text { M. HC double actif }\end{array}$} \\
\hline & Aide & \multicolumn{12}{|c|}{ Weekends + congés } \\
\hline \multirow{2}{*}{$\begin{array}{l}\text { Conduite } \\
\text { technique }\end{array}$} & Troupeau & \multicolumn{12}{|c|}{ Troupeau en stabulation, vêlages étalés, affouragement en vert } \\
\hline & Surfaces & & & & & & & \multicolumn{3}{|c|}{ TSSF } & & & \\
\hline \multirow[t]{2}{*}{ Durées } & TA & \multicolumn{12}{|c|}{$\mathrm{TA}=1494 \mathrm{~h} / \mathrm{an} ; \mathrm{TAcb} / \mathrm{pCB}=1132 \mathrm{~h} / \mathrm{an}$} \\
\hline & TS & \multicolumn{12}{|c|}{$\mathrm{TS}=34$ jours/an } \\
\hline \multirow{4}{*}{$\begin{array}{l}\text { Formes } \\
\text { d'organisation }\end{array}$} & Périodes & \multicolumn{6}{|c|}{1} & \multicolumn{3}{|c|}{2} & \multicolumn{3}{|c|}{3} \\
\hline & $\mathrm{FOO}$ & \multicolumn{6}{|c|}{ FOQ1, FOQ2 } & \multicolumn{3}{|c|}{ FOQ3, FOQ4 } & \multicolumn{3}{|c|}{ FOQ5, FOQ6 } \\
\hline & & \multicolumn{6}{|c|}{ FOO 1 : enfants absents (école) } & \multicolumn{3}{|c|}{$\begin{array}{c}\text { FOQ3 : congés enfants, } \\
\text { TSSF }\end{array}$} & & & \\
\hline & & \multicolumn{6}{|c|}{$\begin{array}{l}\text { Mme HC réalise le travail d'astreinte seule } \\
\text { (nettoyage stabulation, traite, vente du lait, } \\
\text { distribution ration, coupe d'herbe). M. HC } \\
\text { travaille à l'extérieur (vente produits } \\
\text { vétérinaires) }\end{array}$} & \multicolumn{3}{|c|}{$\begin{array}{l}\text { Mlme HC fait la traite } \\
\text { puis vend le lait. Pendant } \\
\text { ce temps, les enfants } \\
\text { nettoient la stabulation } \\
\text { puis donnent à manger } \\
\text { aux vaches. Puis Mme HC } \\
\text { et les enfants coupent } \\
\text { I'herbe. Une fois le travail } \\
\text { d'astreinte terminé, } \\
\text { Mme HC et les enfants } \\
\text { rejoignent M. HC et } \\
\text { les salariés saisonniers } \\
\text { pour les travaux dans } \\
\text { les champs. }\end{array}$} & & & \\
\hline
\end{tabular}

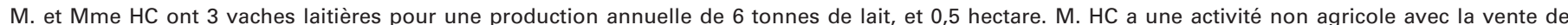

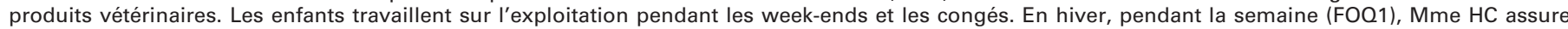

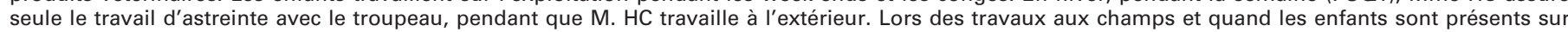

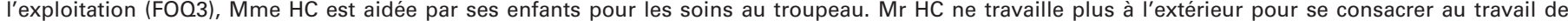
saison avec des salariés saisonniers. Les travaux ne sont pas mécanisés.

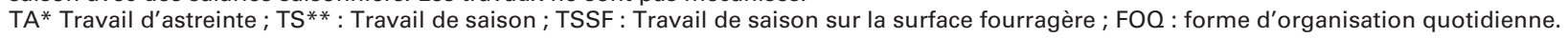

une relation directe entre le travail de saison et la surface : la durée du travail de saison, exprimée en nombre de jours par an, s'accroît linéairement avec la surface. Cependant les plus grandes exploitations présentent une meilleure efficience du travail de saison (nombre de jours de travail de saison [TS] par hectare de SAU) (tableau 5). Dans notre échantillon, les exploitations avec une SAU supérieure à 4 hectares consacrent moins de 37,7 jours par an par hectare, alors que les exploitations dont la SAU est inférieure à 2 hectares y consacrent plus de 62 jours par hectare. Indépendamment de la taille du cheptel et de la surface, pour écrêter les pointes de travail dans les champs (labour, foin, ensilage), tous les éleveurs ont recours au salariat saisonnier et à l'entraide entre voisins. Les salariés permanents, dans les quatre exploitations où ils sont employés, sont également mobilisés pour ces travaux.

\section{Variabilité des formes d'organisation du travail au cours de la campagne}

Pendant l'année, plusieurs formes d'organisation du travail d'astreinte alternent (tableau 2). Les changements sont liés à la disponibilité de la main-d'œuvre, au climat ou encore à la présence d'une activité non agricole (vente de plantes médicinales, etc.). De juin à septembre lors des périodes de travaux dans les champs, les éleveurs réorganisent la main-d'œuvre affectée au travail d'astreinte pendant la journée. Par exemple, M. HC arrête son activité non agricole (vendeur de produits vétérinaires dans un magasin en ville) pour assurer, avec des salariés saisonniers, les travaux dans les champs (tableau 2). Mme HC assure le travail d'astreinte avec le troupeau laitier en déléguant des tâches aux enfants (nettoyage de la stabulation et distribution de l'affouragement). Le reste de 
Tableau 3. Les variables relatives à l'organisation du travail et modalités prises par les cas.

Table 3. Variables to characterize work organisation and modalities of the cases.

\begin{tabular}{|c|c|c|}
\hline Thèmes & Variables & Modalités \\
\hline \multirow[t]{2}{*}{ Durées du travail } & Nb heures de travail d'astreinte (TA) par an & $\begin{array}{l}1 \text { - TA faible (<2 } 500 \text { h/an) } \\
2 \text { - TA moyen (2 } 500 \text { à } 4000 \text { h/an) } \\
3 \text { - TA élevé (plus de } 4000 \text { h/an) }\end{array}$ \\
\hline & Nb de jours de travail de saison (TS) par an & $\begin{array}{l}1 \text { - TS faible < } 75 \text { j/an } \\
2 \text { - TS moyen } 75 \text { à } 150 \text { j/an } \\
3 \text { - TS élevé > } 150 \text { j/an }\end{array}$ \\
\hline \multirow[t]{4}{*}{$\begin{array}{l}\text { Formes d'organisation } \\
\text { du travail (FOQ) }\end{array}$} & Variabilité de I'organisation annuelle du travail & $\begin{array}{l}1 \text { - Organisation du travail stable sur l'année } \\
\text { (une ou deux périodes) } \\
2 \text { - Organisation du travail peu variable sur l'année } \\
\text { (trois périodes) } \\
3 \text { - Organisation du travail variable sur l'année } \\
\text { (> trois périodes) }\end{array}$ \\
\hline & Origine des périodes & $\begin{array}{l}1 \text { - Les périodes sont liées aux interactions TA-TS } \\
2 \text { - Les périodes sont liées au processus technique, } \\
\text { à la main-d'œuvre, au climat } \\
3 \text { - Les périodes sont liées à la combinaison } \\
\text { d'activités non agricoles }\end{array}$ \\
\hline & $\begin{array}{l}\text { Variabilité des formes d'organisation } \\
\text { quotidienne (FOQ) }\end{array}$ & $\begin{array}{l}1 \text { - Pas de variation des } \mathrm{FOQ} \\
\text { (une seule FOQ par période) } \\
2 \text { - Plusieurs FOQ pendant la période de travaux } \\
\text { dans les champs, sinon une seule FOQ } \\
3 \text { - Plusieurs FOQ par période }\end{array}$ \\
\hline & Division du travail d'astreinte & $\begin{array}{l}1 \text { - Le TA est réalisé en autonomie par la cellule } \\
\text { de base } \\
2 \text { - Le TA est partagé avec l'aide bénévole } \\
3 \text { - Le TA est délégué à des salariés permanents }\end{array}$ \\
\hline
\end{tabular}

\section{Tableau 4. Tableau de Bertin et types d'organisation du travail pour les neuf exploitations laitières étudiées.}

Table 4. Bertin's table and forms of work organization for the nine dairy farms studied.

\begin{tabular}{|c|c|c|c|c|c|c|c|c|c|}
\hline & HP & TY & VN & DE & CN & CG & DC & HG & $\mathrm{HC}$ \\
\hline $\mathrm{Nb}$ heures de travail d'astreinte (TA) par an & 3 & 3 & 3 & 3 & 3 & 2 & 2 & 1 & 1 \\
\hline $\mathrm{Nb}$ de jours de travail de saison (TS) par an & 3 & 2 & 3 & 3 & 3 & 2 & 2 & 1 & 1 \\
\hline Variabilité de l'organisation annuelle du travail & 3 & 3 & 3 & 3 & 2 & 2 & 1 & 2 & 1 \\
\hline Origine des périodes & 3 & 3 & 3 & 1 & 1 & 2 & 1 & 1 & 1 \\
\hline Variabilité des formes d'organisation quotidienne (FOQ) & 2 & 2 & 2 & 1 & 1 & 3 & 1 & 3 & 3 \\
\hline Division du travail d'astreinte & 3 & 3 & 3 & 1 & 2 & 2 & 1 & 2 & 2 \\
\hline Groupes & \multicolumn{4}{|c|}{ I } & \multicolumn{2}{|c|}{ II } & \multicolumn{3}{|c|}{ III } \\
\hline
\end{tabular}

l'année, le travail, centré autour du troupeau laitier (traite, alimentation, etc.), est réalisé par Mme pendant que M. se consacre à la double activité. Ces formes d'organisation sont qualifiées de très stables, car l'année est découpée en seulement deux périodes, c'est-à-dire deux modalités de contenu et de réparti- tion du travail d'astreinte entre les personnes durant l'année. Ces deux périodes sont déterminées par des changements du processus technique. Dans les exploitations de notre échantillon, il n'apparaît pas de relation entre la taille du troupeau et les formes d'organisation du travail d'astreinte.

\section{Types d'organisation du travail}

Trois types d'organisation du travail ont été identifiés, en fonction des types d'exploitations, à partir des données sur les durées et les formes d'organisation du travail d'astreinte (tableau 4). 
Tableau 5. Durées du travail dans les neuf exploitations laitières.

Table 5. Work duration in the nine dairy farms.

\begin{tabular}{lccccccrrr}
\hline Exploitation & $\begin{array}{c}\text { TA total } \\
\text { (h/ha) }\end{array}$ & $\begin{array}{c}\text { TA de } \\
\text { la cellule } \\
\text { de base } \\
\text { (h/an) }\end{array}$ & $\begin{array}{c}\text { TA hors } \\
\text { cellule } \\
\text { de base } \\
\text { (\%) }\end{array}$ & $\begin{array}{c}\text { TA/vache } \\
\text { (heure/an) }\end{array}$ & $\begin{array}{c}\text { Nombre } \\
\text { de vaches }\end{array}$ & Mécanisation & $\begin{array}{c}\text { TS } \\
\text { (j/an) }\end{array}$ & $\begin{array}{c}\text { TS/ha } \\
\text { Surface } \\
\text { (ha) }\end{array}$ \\
\hline HP & 4722 & 2713 & 43 & 236 & 20 & Traite et coupe herbe & 199 & 28,4 & 7 \\
TY & 3943 & 2399 & 39 & 563 & 7 & Traite et coupe herbe & 102 & 51 & 2 \\
VN & 6024 & 2191 & 65 & 317 & 19 & Traite et coupe herbe & 177 & 37,7 & 4,7 \\
DE & 4823 & 2336 & 52 & 482 & 10 & Traite & 150 & 37,5 & 4 \\
CN & 4056 & 3912 & 4 & 507 & 8 & Traite et coupe herbe & 138 & 69 & 2 \\
CG & 3306 & 2715 & 18 & 472 & 7 & Traite et coupe herbe & 155 & 45,6 & 3,4 \\
DC & 2715 & 2715 & 0 & 543 & 5 & Non & 122 & 67,8 & 1,8 \\
HG & 2264 & 2264 & 0 & 566 & 4 & Non & 75 & 62,5 & 1,2 \\
HC & 1494 & 1237 & 17 & 498 & 3 & Non & 34 & 68 & 0,5 \\
\hline
\end{tabular}

TA : travail d'astreinte; TS : travail de saison.

Grands mécanisés avec salariat permanent (type I)

Dans quatre exploitations, la durée du travail d'astreinte est élevée avec plus de 4000 heures par an. Pour alléger sa charge en travail, la cellule de base délègue une part importante du travail d'astreinte (coupe d'herbe et/ou traite) à un salarié permanent et à des aides bénévoles. Par exemple, en semaine, le travail est partagé entre la cellule de base et le salarié permanent. Les week-ends, la cellule de base compte également sur l'aide des enfants et leur confie certaines tâches. Le recours à la mécanisation (pot trayeur et rotofil) permet d'améliorer l'efficience du travail d'astreinte (236 à 482 heures de TA/vache). Si la durée du travail de saison sur la surface fourragère est élevée (plus de 150 jours par an), le travail est également le plus efficient de notre échantillon (28,4 à 51 jours/ha de SAU). Dans trois exploitations, les formes d'organisation du travail d'astreinte sont caractérisées par leur forte variabilité au cours de l'année pour s'adapter au climat, gérer les pointes de travail avec les surfaces, et répondre aux attentes de temps libre des exploitants (congés). En été, lors des pointes de travail avec la surface fourragère (récoltes, etc.), le salarié permanent se consacre aux foins et n'intervient plus avec le troupeau. La cellule de base assure alors seule le travail d'astreinte. Le salarié remplace les exploitants également pendant leurs congés. Dans une exploitation (DE), l'organisation du travail dans l'année est plus stable. En hiver, il y a une seule forme d'organisation du travail d'astreinte où la cellule de base (le couple) et le salarié permanent se partagent les travaux d'astreinte. En été, quand les travaux de saison (labour, récoltes, etc.) deviennent prioritaires, la cellule de base réalise le travail d'astreinte alors que le salarié travaille au champ toute la journée. Il n'y a pas de recours au bénévolat familial dans cette exploitation. Les quatre exploitations sont de grande taille ( 7 à 20 vaches, 2 à 7 hectares) et spécialisées en lait. Elles présentent un niveau d'intensification plutôt élevé en termes de productivité par vache (plus de 5000 litres de lait/vache/an).

Mécanisés avec l'aide des enfants ajustant les formes d'organisation du travail d'astreinte (type II)

Le travail d'astreinte (de 3300 à 4000 heures/an) est réalisé en quasi-autonomie par la cellule de base (le couple), avec l'aide des enfants les week-ends. Même si les exploitants ont recours à la mécanisation (traite et coupe d'herbe), l'efficience du travail d'astreinte est moindre (472 à 507 heures de TA/vache/an). Pour réaliser le volume élevé de travail de saison avec la surface fourragère (138 jours/an), la cellule de base a recours à l'emploi de salariés saisonniers, l'aide bénévole des enfants et l'entraide avec les voisins. L'efficience du travail de saison est moyenne (45 à 69 jours de TS/ha de SAU). Dans une exploitation (CG), plusieurs périodes existent en fonction des priorités entre travaux de saison, travail d'astreinte et activité non agricole. $\mathrm{Au}$ sein de ces périodes, plusieurs formes d'organisation du travail d'astreinte alter- nent selon le rythme hebdomadaire des enfants. En effet, ceux-ci aident leurs parents pour le troupeau laitier pendant les week-ends. Dans l'exploitation CN, la forme d'organisation du travail d'astreinte est stable. Les périodes sont rythmées par le processus de production. Ces deux exploitations de taille moyenne (5 à 7 vaches, 3 hectares) ont un niveau de productivité par vache relativement élevé (4300 à 5000 litres de lait/vache/an).

\section{Petits non mécanisés}

ajustant l'activité non agricole si elle existe (type III)

Dans les trois exploitations, la durée du travail d'astreinte est inférieure à 2300 heures par an. Le travail d'astreinte est réalisé en autonomie par la cellule de base ou partagé avec les enfants. Il est peu efficient, avec en moyenne 530 heures de TA/vache/an. Ces exploitations n'ont pas recours à la mécanisation. Le travail de saison est partagé entre la cellule de base, les enfants et des salariés saisonniers. Ces exploitations consacrent le plus de jours de TS par ha de SAU (66 journées/hectare). Dans deux exploitations, l'ajustement de l'activité non agricole aux charges de travail d'élevage marque les changements de formes d'organisation du travail d'astreinte. En effet, l'activité non agricole est arrêtée lorsqu'il y a concurrence entre travaux d'astreinte et de saison afin que la cellule de base puisse se consacrer aux travaux agricoles. L'emploi de salariés temporaires sert également à passer les pointes de travail. Ces exploitations sont les plus petites de 
l'échantillon (moins de 5 têtes et moins de 1,8 ha). Elles ont également le plus faible niveau d'intensification (2 000 litres de lait/vache/an dans deux élevages).

\section{Discussion}

L'échantillon d'exploitations étudiées n'est bien sûr pas représentatif de l'ensemble des exploitations laitières vietnamiennes, mais donne des tendances sur les implications d'un accroissement de la taille des cheptels sur l'organisation du travail dans la région de Moc Chau. L'agrandissement des troupeaux laitiers induit des durées du travail d'astreinte et de saison plus élevées. Il s'accompagne d'une amélioration de l'efficience du travail d'astreinte par vache laitière et du travail de saison par hectare de SAU. L'hypothèse d'une rationalisation du travail par l'accroissement de la taille du cheptel est donc partiellement vérifiée. Si l'accroissement des troupeaux laitiers semble s'accompagner d'une amélioration de la productivité par vache, il serait nécessaire de valider cette hypothèse en conduisant un dispositif de recherche auprès d'un échantillon plus grand d'exploitations laitières, et en mesurant les performances animales. En effet, d'autres facteurs non pris en compte dans notre étude peuvent influencer le niveau de production des cheptels: potentiel génétique, qualité des aliments distribués, sanitaire.

Nos résultats soulignent également que les éleveurs disposent de différents leviers pour s'adapter aux pointes de travail qui sont le recours à de la main-d'œuvre extérieure (salarié, aide bénévole, entraide entre voisins), ou la modulation des activités non agricoles. Les plus grandes exploitations, qui dans notre échantillon sont spécialisées, ont recours à l'emploi de salariés permanents. Les plus petites exploitations, associant la production laitière à d'autres activités non agricoles, arrêtent la double activité pour accroittre temporairement leur disponibilité en main-d'œuvre familiale. L'organisation du travail d'astreinte lors des périodes de travail dans les champs est ainsi modifiée : le travail d'astreinte est partagé entre les travailleurs de la famille ou le salarié se consacre temporairement au travail de saison. Ainsi, sans remettre en cause le caractère familial des exploitations, l'accroissement de la taille des cheptels s'accompagne d'un recours significatif au salariat. Si ce recours au salariat rural n'est pas une solution nouvelle, il n'est pas forcément associé aux voies possibles d'évolution de l'exploitation familiale. Il reste peu connu et ouvre un vaste débat sur les voies à développer pour assurer un emploi décent en milieu rural (Madelrieux et al., 2009).

Les durées du travail sont élevées dans ces exploitations de petites dimensions (Hostiou et Dedieu, 2009). Ces durées s'expliquent par un modèle technique moderne, finalement très proche de ceux proposés en France, mais associé à un très faible niveau de mécanisation. De plus, du fait d'un itinéraire technique fortement encadré et normé par la coopérative, les éleveurs ne semblent pas simplifier les conduites techniques d'élevage pour réduire la durée du travail, contrairement à ce qui se passe dans d'autres situations d'exploitations familiales (Hostiou et Dedieu, 2009 ; Hostiou et Fagon, 2009). Cependant, des études ont montré que, même dans les systèmes industriels très normalisés comme l'élevage de porcs, les éleveurs peuvent simplifier leurs protocoles de surveillance ou d'intervention sur les animaux (Martel et al., 2008). Un dispositif de recueil des pratiques d'élevage d'éleveurs laitiers vietnamiens pourrait être mis en place, par exemple avec des suivis d'élevage, pour vérifier l'hypothèse selon laquelle des simplifications ponctuelles sont opérées par les éleveurs et pour analyser leurs effets sur les performances animales. Par ailleurs, les importants volumes de travail, la pénibilité de tâches manuelles, associée à la faible rémunération du travail agricole, peuvent expliquer une certaine désaffection pour ce métier des descendants, qui préfèrent travailler en ville.

Nos résultats nous amènent à proposer quelques pistes pour accompagner le développement de l'activité laitière à Moc Chau. La coopérative pourrait mettre en place des mesures pour maintenir un tissu d'exploitations laitières diverses en taille, en main-d'œuvre et en combinaisons d'activités agricoles et non agricoles. En effet, les voies de résolution des problèmes liés au travail des éleveurs ne se présentent pas de la même manière pour toutes les exploitations, comme nos résultats le montrent. Pour les plus petites exploitations et/ou avec de faibles capacités financières, il faudrait pouvoir développer une mécanisation en commun, si cette formule coopérative n'est pas socialement incompatible avec l'histoire de la collectivisation et le récent processus de privatisation des fermes d'État.

\section{Conclusion}

Notre étude explore la question du travail en élevage laitier familial en identifiant certaines relations entre taille du troupeau, durées et formes d'organisation du travail, dans un contexte où la rationalisation du travail par l'agrandissement des cheptels pourrait être une voie d'amélioration des volumes de production à l'échelle nationale. Nous montrons que les éleveurs laitiers disposent de plusieurs leviers pour organiser leur travail. Les cas d'agrandissement étudiés s'appuient sur la mécanisation du travail d'astreinte avec le troupeau ainsi que sa délégation à des salariés permanents, permettant d'en améliorer l'efficience. Les plus petites structures, quant à elles, ajustent la part non agricole de leur activité. Dans toutes les exploitations, indépendamment de la taille, les pointes de travail aux champs imposent des modifications de la répartition des tâches, l'embauche de saisonniers et l'entraide entre voisins. Le développement de la production laitière pourrait reposer sur le maintien de cette diversité d'exploitations avec la mise en place de mesures qui traiteraient différemment, selon les cas, les problèmes d'accès à la main-d'œuvre et à la mécanisation. La prise en considération des conditions de travail des éleveurs en milieu rural est également un enjeu pour développer des systèmes de production durables et vivables.

\section{Remerciements}

L'étude a bénéficié d'un financement de l'Agence nationale de la recherche (ANR). Cette étude avait également comme objectif de former un chercheur vietnamien aux méthodes d'analyse sur le travail développées par des zootechniciens des systèmes d'élevage français. Elle a débouché sur l'organisation d'une formation des partenaires à l'approche globale des exploitations, incluant la dimension travail (Hostiou, 2008). Les auteurs remercient les éleveurs qui ont participé à cette étude.

\section{Références}

Bertin J. La graphique et le traitement graphique de l'information. Paris : Flammarion, 1977.

Cramb RA, Purcell T, Ho TCS. Participatory assessment of rural livelihoods in the Central Highlands of Vietnam. Agric Syst 2004; 81 : 255-72. 
Dedieu B, Servière G, Madelrieux S, Dobremez L, Cournut S. Comment appréhender conjointement les changements techniques et les changements du travail en élevage? Cah Agric 2006 15 : 506-13. DOI : 10.1684/agr.2006.0028

Girard N, Bellon S, Hubert B, Lardon S, Moulin $\mathrm{CH}$, Osty PL. Categorising combinations of farmers' land use practices: an approach based on examples of sheep farms in the south of France. Agronomie 2001 ; 21 : 435-59.

Hostiou N, Dedieu B. Diversity of forage system work and adoption of intensive techniques in dairy cattle farms of Amazonia. Agron Sustain Dev 2009 ; 29 : 535-44.
Hostiou N, Fagon J. Simplification des conduites d'élevage: analyse transversale des techniques mises en œuvre dans les filières herbivores et granivores. Actes du colloque "Rencontres travail en élevage », Rennes, 2009.

Hostiou N. Formation sur les systèmes d'élevage. Hanoi : Rudec; Prise, 2008 (cédérom).

Madelrieux S, Dedieu B. Qualification and assessment of work organisation in livestock farms. Animal 2008 ; 2 : 435-46.

Madelrieux S, Dupré L, Rémy J. Itinéraires croisés et relations entre éleveurs et salariés dans les Alpes du Nord. Economie Rurale 2009 ; (313-314) : 6-21.
Martel G, Dourmad JY, Dedieu B. Do labour productivity and preferences about work load distribution affect reproduction management and performance in pig farms? Livestock Science 2008 118: 96-117.

Martin C, Castella JC, Anh HL, Eguienta Y, Hieu TT. A participatory simulation to facilitate farmer's adoption of livestock feeding systems based on conservation agriculture in the Uplands of Northern Vietnam. International Journal of Agricultural Sustainability 2004; 2 : 118-32.

Suzuki K, Kanameda M, Ogawa T, et al. Productivity and socio-economic profile of dairy cattle farmers amongst rural smallholder communities in northern Vietnam. Livest Sci 2006; 101 242-50. 